Supporting Information

\title{
Cell-selective Messenger RNA Delivery and CRISPR/Cas9 Genome Editing by Modulating the Interface of Phenylboronic Acid-derived Lipid Nanoparticles and Cellular Surface Sialic Acid
}

\author{
Qiao Tang, ${ }^{\dagger,+}$ Ji Liu, ${ }^{\dagger, \xi}$ Ying Jiang, ${ }^{ \pm}$Meining Zhang, ${ }^{+}$Lanqun Mao ${ }^{\dagger}, \xi$ and Ming Wang ${ }^{*}, \xi$
}

${ }^{\dagger}$ Beijing National Laboratory for Molecular Sciences, Key Laboratory of Analytical Chemistry for

Living Biosystems, Institute of Chemistry Chinese Academy of Sciences, Beijing 100190, China

* Department of Chemistry, Renmin University of China, Beijing 100872, China

${ }^{ \pm}$College of Chemistry, Beijing Normal University, Beijing 100875, China

$\S$ University of Chinese Academy of Sciences, Beijing 100049, China

*Correspondence to: mingwang@iccas.ac.cn

Email: mingwang@iccas.ac.cn 


\section{General}

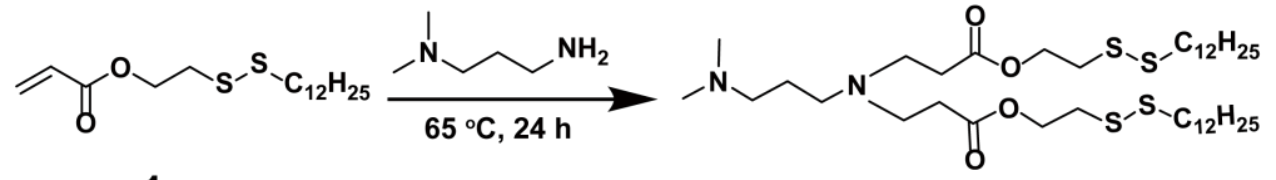

1

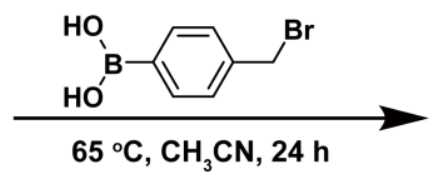

BADP

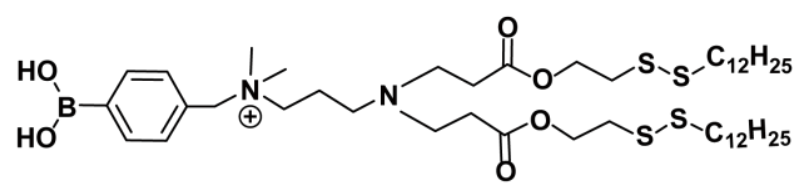

PBA-BADP

Scheme S1. Synthesis route of PBA-BADP lipid.

Table S1. Sequences of PCR primers for amplifying target region and sequences of sgRNAs targeting GFP and HPV18E6 gene.

\begin{tabular}{c|c}
\hline Oligo name & Sequence (5' - 3') \\
\hline sgGFP & GGGCACGGGCAGCTTGCCGG \\
sgHPV18E6 & GGGCGCTTTGAGGATCCAACA \\
GFP-Forward & ATCTCGAATATGGTGAGCAAGGGCG \\
GFP-Reverse & GTCCATGCCGAGAGTGATCC \\
HPV18E6-Forward & GGGAGTGACCGAAAACGGTC \\
HPV18E6-Reverse & ATTCAACGGTTTCTGGCAC \\
\hline
\end{tabular}


Table S2. Z-average hydrodynamic size and zeta potential of PBA-BADP/mRNA and BADP/mRNA nanoparticles was measured by dynamic light scattering and electrophoretic light scattering; data was given by the instrument with triplicate readouts.

\begin{tabular}{c|c|c}
\hline NPs & $\begin{array}{c}\text { Z-average } \\
\text { hydrodynamic size/ nm }\end{array}$ & $\begin{array}{c}\text { Zeta potential/ } \\
\mathrm{mV}\end{array}$ \\
\hline PBA-BADP+Luci mRNA & $64 \pm 2$ & $24.1 \pm 1.0$ \\
PBA-BADP+p53 mRNA & $77 \pm 3$ & $14.6 \pm 2.0$ \\
PBA-BADP+Cas9 mRNA/sgRNA & $111 \pm 2$ & $23.9 \pm 0.9$ \\
BADP+Luci mRNA & $207 \pm 4$ & $35.7 \pm 1.0$ \\
BADP+p53 mRNA & $168 \pm 3$ & $36.1 \pm 2.2$ \\
BADP+Cas9 mRNA/sgRNA & $241 \pm 3$ & $27.3 \pm 0.7$ \\
\hline
\end{tabular}

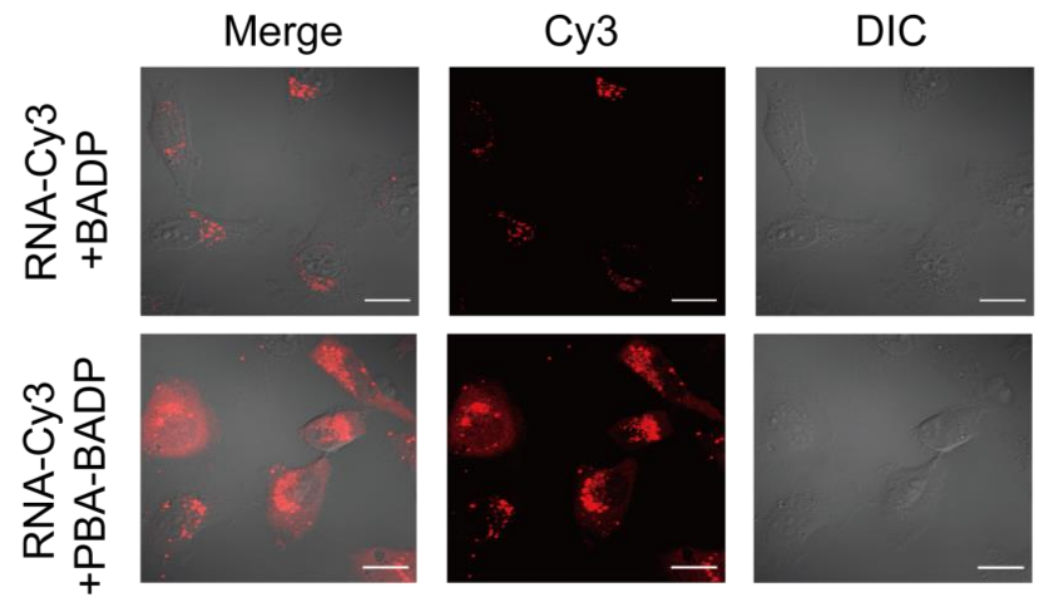

Figure S1. CLSM image of HeLa cells treated with $10 \mathrm{nM}$ Cy3-RNA using PBA-BADP and BADP for 8 h. Scale bar: $20 \mu \mathrm{m}$. 


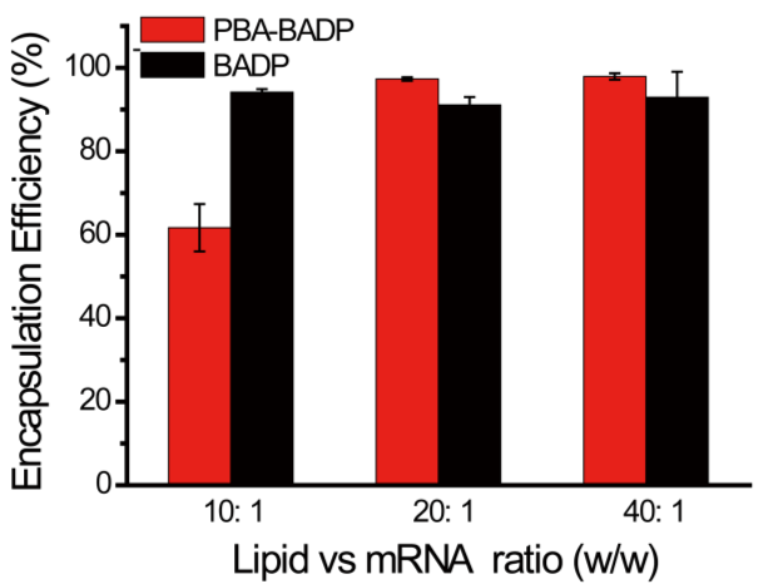

Figure S2. Comparison of mRNA encapsulation efficiency by PBA-BADP or BADP NPs. 200 ng Luci mRNA was mixed with either PBA-BADP or BADP at different mass ratio of lipid in phosphate buffer solutions $(\mathrm{pH}=7.2,10 \mathrm{mM})$, followed by RNA concentration determination using iQuant broad range RNA quantification kit.

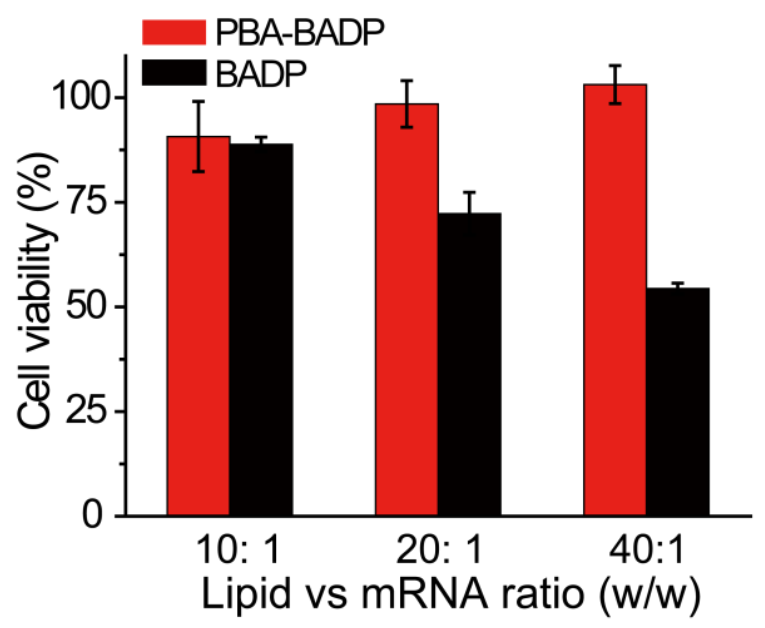

Figure S3. The viability of HeLa cells treated with PBA-BADP/Luci mRNA and BADP/Luci mRNA NPs prepared at indicated weight ratios. $330 \mathrm{ng} / \mathrm{mL}$ mRNA was delivered into cells using PBA-BADP or BADP NPs. 


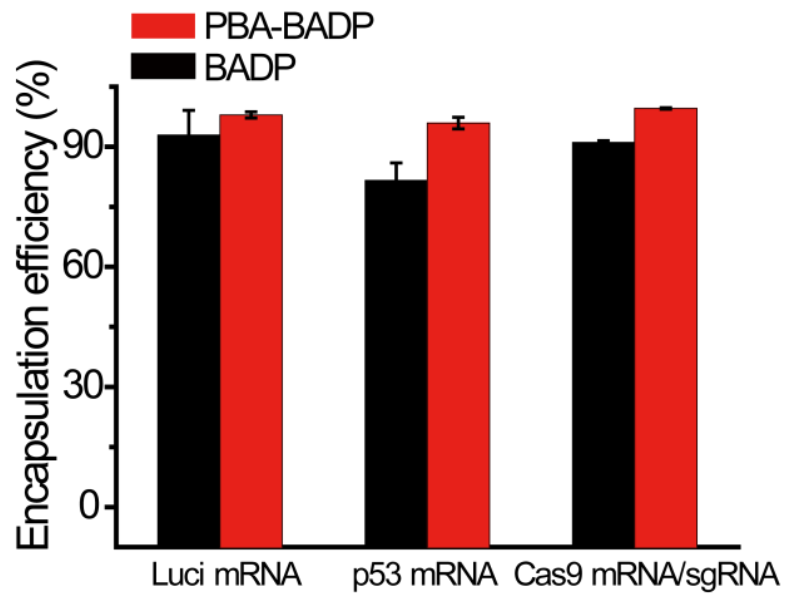

Figure S4. Comparison of the encapsulation efficiency of mRNA by PBA-BADP or BADP NPs. 200 ng different mRNA was mixed with PBA-BADP or BADP NPs at weight ratios of lipid: $\mathrm{mRNA}=40: 1$ in phosphate buffer solutions ( $\mathrm{pH}=7.2,10 \mathrm{mM}$ ), followed by RNA concentration determination using iQuant broad range RNA quantification kit.

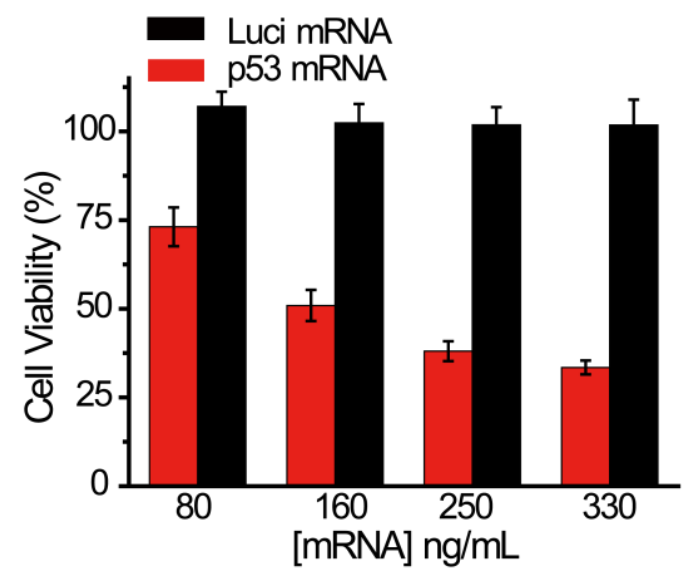

Figure S5. The viability of SiHa cells treated with increased concentration of PBA-BADP/p53 mRNA or PBA-BADP/Luci mRNA NPs at optimized condition. 


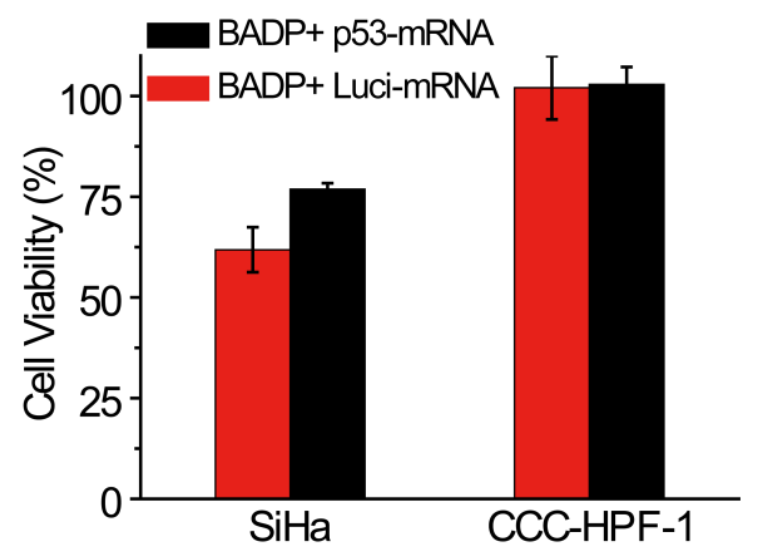

Figure S6. The viability of SiHa (cancer cells) and CCC-HPF-1 (normal cells) treated with BADP/p53 mRNA or BADP/Luci mRNA NPs containing $330 \mathrm{ng} / \mathrm{mL}$ mRNA.

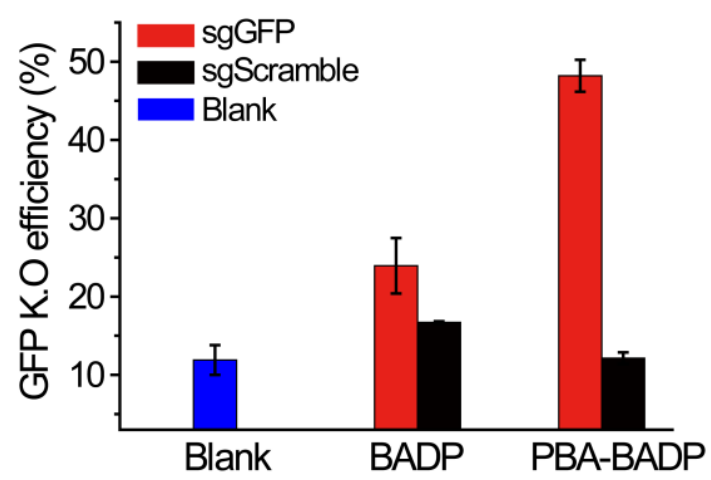

Figure S7. GFP knockout efficiency of GFP-HeLa cells treated with Cas9 mRNA/sgRNA complexed with BADP or PBA-BADP NPs (containing $330 \mathrm{ng} / \mathrm{mL}$ Cas9 mRNA and $13.3 \mathrm{nM}$ sgRNA). 
(a)

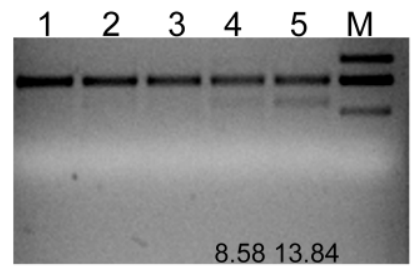

(b)

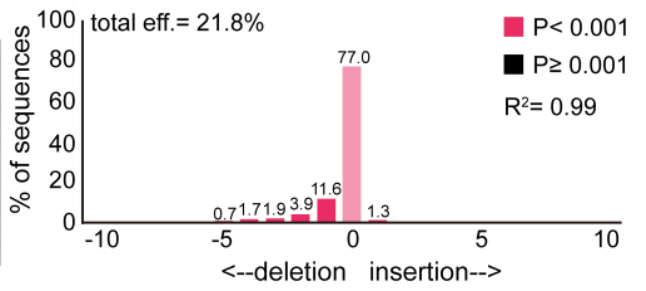

(c)

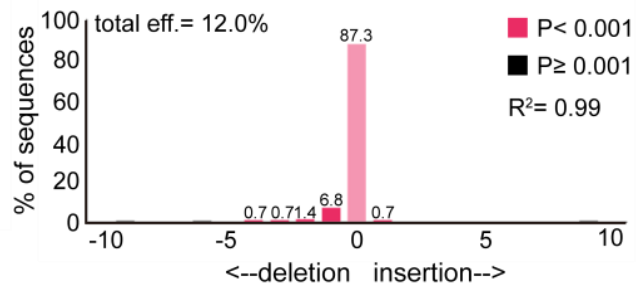

Figure S8. T7E1 assay and DNA sequencing analysis of GFP-HeLa cells with different Cas9 mRNA NPs treatments. (a) T7E1 assay of the indel efficiency of PCR products of the targeted GFP site of GFPHeLa cells with different lipid/Cas9 mRNA/sgRNA NPs treatments (containing $330 \mathrm{ng} / \mathrm{mL}$ Cas9 mRNA and 13.3 nM sgRNA). Lane 1: blank; lane 2: BADP/Cas9 mRNA/sgScramble; lane 3: PBABADP/Cas9 mRNA/sgScramble; lane 4: BADP/Cas9 mRNA/sgGFP; lane 5: PBA-BADP/Cas9 mRNA/sgGFP. DNA sequencing results of PCR products of the targeted GFP sites of GFP-HeLa cells treated with PBA-BADP/Cas9 mRNA/sgGFP NPs (b) or BADP/Cas9 mRNA/sgGFP NPs (c).

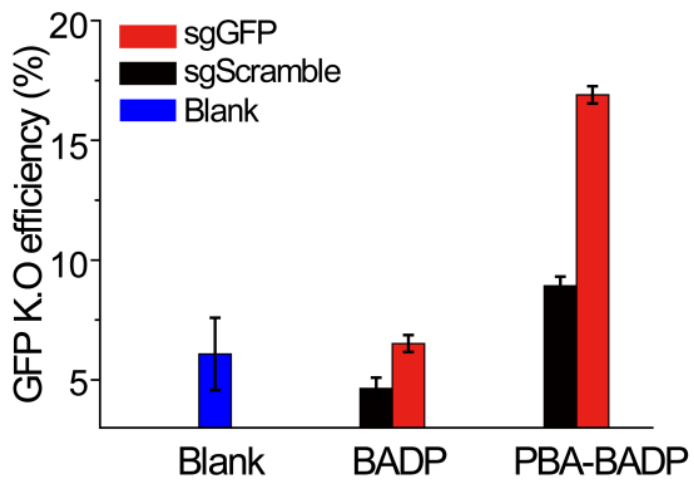

Figure S9. GFP knockout efficiency of GFP-HEK293 cells treated with Cas9 mRNA/sgRNA complexed with BADP or PBA-BADP NPs (containing $330 \mathrm{ng} / \mathrm{mL}$ Cas9 mRNA and $13.3 \mathrm{nM}$ sgRNA). 
(a)

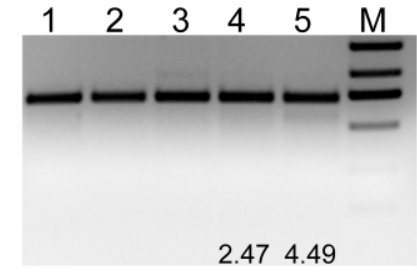

(b)

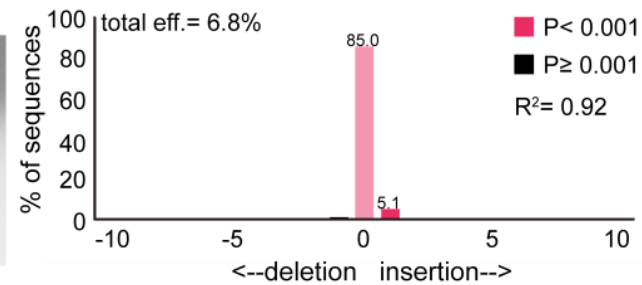

(c)

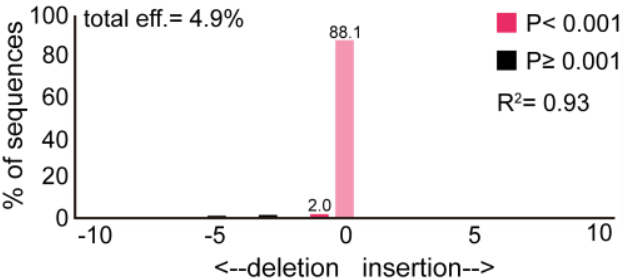

Figure S10. T7E1 assay and DNA sequencing analysis of GFP-HEK293 cells with different Cas9 mRNA NPs treatments. (a) T7E1 assay of the indel efficiency of PCR products of the targeted GFP site of GFP-HEK293 cells with different lipid/Cas9 mRNA/sgRNA NPs treatments (containing $330 \mathrm{ng} / \mathrm{mL}$ Cas9 mRNA and $13.3 \mathrm{nM}$ sgRNA). Lane 1: blank; lane 2: BADP/Cas9 mRNA/sgScramble; lane 3: PBA-BADP/Cas9 mRNA/sgScramble; lane 4: BADP/Cas9 mRNA/sgGFP; lane 5: PBA-BADP/Cas9 mRNA/sgGFP. DNA sequencing results of PCR products of the targeted GFP sites of GFP-HEK293 cells treated with PBA-BADP/Cas9 mRNA/sgGFP NPs (b) or BADP/Cas9 mRNA/sgGFP NPs (c).

(a)

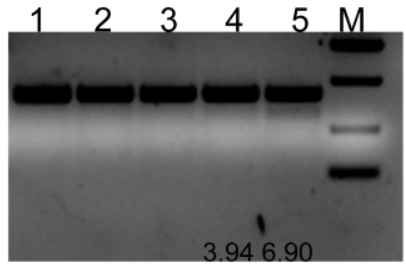

(b)

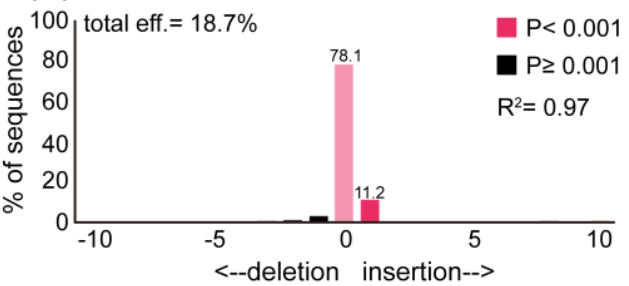

(c)

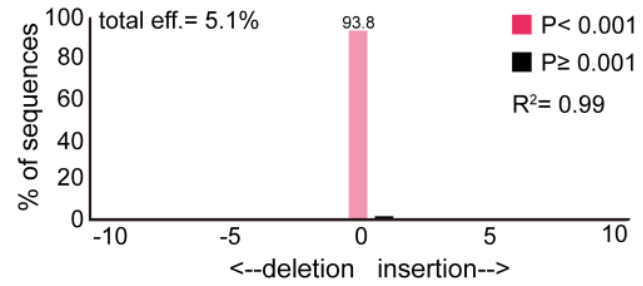

Figure S11. T7E1 assay and DNA sequencing analysis of HeLa cells with different Cas9 mRNA NPs treatments. (a) T7E1 assay of the indel efficiency of PCR products of the targeted HPV18E6 site of HeLa cells with different lipid/Cas9 mRNA/sgRNA NPs treatments (containing $660 \mathrm{ng} / \mathrm{mL}$ Cas9 mRNA and $40 \mathrm{nM}$ sgRNA). Lane 1: blank; lane 2: BADP/Cas9 mRNA/sgScramble; lane 3: PBABADP/Cas9 mRNA/sgScramble; lane 4: BADP/Cas9 mRNA/sgHPV18E6; lane 5: PBA-BADP/Cas9 mRNA/sgHPV18E6. DNA sequencing results of PCR products of the targeted HPV18E6 sites of HeLa cells treated with PBA-BADP/Cas9 mRNA/sgHPV18E6 NPs (b) or BADP/Cas9 mRNA/sgHPV18E6 NPs (c). 


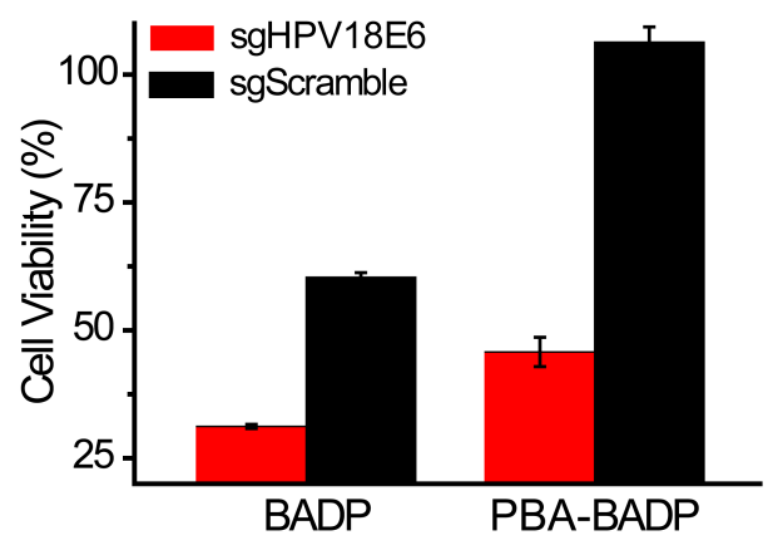

Figure S12. The viability of HeLa cells treated with Cas9 mRNA/sgHPV18E6 complexed with PBABADP or BADP NPs (containing $660 \mathrm{ng} / \mathrm{mL}$ Cas9 mRNA and $40 \mathrm{nM}$ sgRNA). 